\section{Complexity in Daily Living of Older Adults with Multi- morbidity: Health, Social and Informal Care Utilization and Costs \\ Karin Josefsson ${ }^{1 *}$ and Martina Summer Meranius ${ }^{2}$}

${ }^{1}$ Faculty of Caring Science, Work Life and Social Welfare, University of Borås, Sweden

${ }^{2}$ School of Health, Mälardalen University, Västerås, Sweden

\begin{abstract}
Aim: The aim of the study was to describe health, social and informal care utilization and costs for older adults with multimorbidity. The design was descriptive and retrospective.

Methods: The setting was a medium-sized town in an urban area of Sweden and included 10 health centers. Data were collected during 2011 using individual, structured interviews with the informal carers of 20 older adults with multimorbidity. Retrospectively, for a period of 18 months, data were also collected from the older adults' patient registers and records, as data regarding the costs of their health and social care, in- and out-patient care and municipal care including home services.
\end{abstract}

Results: The primary result was that older adults with multimorbidity utilize health and social care from different principals, through different contacts. The results also provide insight into the complexity of these older adults' daily living. Their 18-month health and social care costs varied between 12,084 and 137,187 Euros. For 12 older adults who utilized informal care, their calculated costs varied between 2,092 and 70,590 Euros.

Conclusion: The conclusion is that the increasing number of older adults with multimorbidity and their health and social care utilization and costs should be taken into account in healthcare policy and the organization of health and social care.

*Corresponding author: Karin Josefsson, Faculty of Caring Science, Work Life and Social Welfare, University of Borås, SE 50190 Borås, Sweden, Tel: +46 706689416; E-mail: karin.josefsson@hb.se

Citation: Josefsson K, Meranius MS (2018) Complexity in Daily Living of Older Adults with Multimorbidity: Health, Social and Informal Care Utilization and Costs. J Gerontol Geriatr Med 4: 017.

Received: January 06, 2018; Accepted: February 05, 2018; Published: February 19,2018

\section{Introduction}

By the year 2050, it is estimated that $21 \%$ of the global population will be adults aged 60 years or older [1]. Slightly more than $5 \%$ of the current population of Sweden is 80 years or older [2]. This means that Sweden, along with Italy, has Europe's largest national proportion of older adults. An increasing number of older adults with multimorbidity have been reported $[3,4]$. Longitudinal study showed multimorbidity affects more than half of those over 65 years and more than $75 \%$ of those between 80 and 84 years old [5]. Multimorbidity among adults aged 65 years or older ranges from 55 to $98 \%$ [6]. There are various ways of defining older adults with multimorbidity. In Sweden, one commonly used definition is any adult aged 75 or older who has been hospitalized three or more times in the previous year and has three or more diagnoses (a minimum of three diagnostic codes in their record). This definition is in accordance with the Swedish version of the International Statistical Classification of Diseases and Related Health Problems ICD-10-SE [7].

Multimorbidity correlates with greater use of institutionalized health care, higher health care costs and higher associated costs across the health care system included social care, yet few data exist regarding the associations between multimorbidity and healthcare utilization or cost $[2,4,6,8,9]$. Multimorbidity is common in primary care and in Sweden primary care accounts for the largest cost increase, with a greater need for general medical care and physiotherapy than in previous years $[2,10]$.

The health costs for older adults tend to increase dramatically with the number and combination of comorbidities [5]. Regarding associated costs, little is known about the time spent on specific health-related activities by informal carers who assist older adults with chronic illness [9]. By informal carers means a relative, partner, friend or neighbor who provides care without association with a formal service system. Most (96\%) of informal carers aged 50 years or older have chronic illness themselves and those with greater chronic illnesses feel the greatest demands, including dedicating from 8.5 to 10 hours per day to health-related caring activities. High levels of informal care responsibility have been associated with poorer health. If these informal carers cannot cope with their situation, the health and social care systems face collapse [11].

Sweden is divided into 290 municipalities and 20 county councils including hospital and health care centres [2]. There is no hierarchical relation between county councils and municipalities because they each have their own self-governing local authorities with responsibility for different activities. There is a mix of publicly and privately owned health care facilities but they are generally publicly funded. Ageing in Sweden has been uniquely shaped by a long tradition of locally controlled services for older adults [12]. Responsibility for older (65 years and older) adults' health, social care and home and special accommodation services rests with Sweden's 290 municipalities. Their responsibilities also include providing social support and social services for those released from hospital care [12]. Social 
care is defined as a variety of supportive services delivered in community settings or in a person's home. These services are designed to help older adults to remain living at home, such as personal care with bathing, chore assistance, adult day services and transportation to medical appointments and home-delivered meals. Most health care is provided in health centres by a variety of health professionals such as physicians, district and registered nurses, physiotherapists and occupational therapists. Sweden's hospitals provide specialist care and around-the-clock emergency services. Eight of these are regional hospitals offering highly specialized care [2].

Frustrations with the health system have been reported by older adults with multimorbidity and their relatives, as well as care staff, physicians and health care policymakers $[13,14]$. There are many common challenges in managing multimorbidity, such as a lack of decision-making support, poor communication and uncoordinated health services. Older adults in Sweden with multimorbidity, their relatives, care staff and health care policy makers express problems, such as fragmentation and discontinuity in health and social care management [13]. The continuity was crucial for older adults with multimorbidity, their relatives, care staff and health care policy makers proposed that different forms of cooperation could include common planning of health and social care to create a core group of care staff, thereby reducing the number of different care staff patients were required to meet [12].

There is a need for a multi-perspective approach to health and social care management for older adults with multimorbidity that emphasizes political steering, leadership, co-operation, competence, support for relatives and availability and continuity [13]. Health care providers need to support day-to-day priority-setting and decision-making to improve health outcomes for older adults with multimorbidity [15]. Health, according to Nordenfelt, is when there is a balance between a person's ability to act and his or her goals for action [16]. Taking this into account, it is crucial to describe health, social care utilization, including informal caregiving and costs for older adults with multimorbidity. Despite this, limited research has described the health and social care costs to county councils and communities of older adults with multimorbidity.

\section{Aim}

The aim of this study was to describe health, social and informal care utilization and costs for older adults with multimorbidity.

\section{Research question}

How are the utilization and costs of health, social and informal care for older adults with multimorbidity?

\section{Methods}

\section{Design}

The design was descriptive and retrospective [17]. A descriptive design was used since it intends to describe the actual situation without the necessity of cause and effect analysis. A retrospective survey was used because it provides information about the prevalence, distribution and interrelations of variables within a population [17] The study was performed in 2011 using individual, structured interviews with informal carers for older adults with multimorbidity, as well as 18 months of retrospective data collected from the older adults' patient register, patient records and costs of health and social care, in-patient care, out-patient care and municipal care, including home services. Informal carers are defined as a relative, partner, friend or neighbor who provides care without association with a formal service system. By social care means supportive services delivered in community settings or in a person's home, such as personal care with bathing, chore assistance, adult day services and transportation to medical appointments and home-delivered meals.

\section{Setting and sample}

The study setting was a medium-sized town in an urban area of Sweden, with 14 health centres and one hospital providing specialist care, including around-the-clock emergency services. Ten of the largest health centres were included in the study. Inclusion criteria for older adults (those aged 75 years or older) with multimorbidity included being hospitalized three or more times in the previous three months and having three or more diagnoses (a minimum of three diagnostic codes in their record) according to the Swedish version of the International Statistical Classification of Diseases and Related Health Problems ICD-10-SE [7]. An exclusion criterion was living in special housing for older adults, since there is around the clock nursing care. The sample was selected using the registry systems at the local hospital.

In total, 249 older adults met the inclusion criteria and $10 \%$ of those were randomly selected, with both sexes represented and invited to participate. Of the 25 older adults invited, 20 participated. The remaining five did not participate due to hospitalization or transfer to a nursing home, or for unstated reasons. First, the participants were contacted by phone by one of the investigators, who informed them about the study and asked for permission to send them a letter with more study information to be read in their own time. Next, the same investigator who called initially called again and asked if they were interested in participating. After that, the investigator made a home visit and obtained written informed consent to access the participant's patient register and records. The participants were asked if they had a main relative who helped them at home with their care and was familiar with their situation. Twelve had an informal carer. These participants gave their permission for the investigator to contact their informal carer and for that individual to provide data about them.

Second, informal carers were contacted by phone after receiving a letter informing them about the study. All agreed to participate, and their informed consent was obtained.

\section{Data collection}

Informal carers were interviewed separately by phone at home for 30-60 minutes about the participants' utilization of health and social care [17]. The questions were constructed based and after discussing an initial draft at a seminar with colleagues and project group members, including economists. Two main question sections were developed to meet the study aims [18]. The first section asked about background characteristics such as relationship with the participant, whether they lived together, age, gender, and whether they worked or were retired. The second section consisted of questions about their efforts to help the participant in terms of approximate time spent in categories including cleaning, grocery shopping, washing, cooking, showering and or bathing, dressing, visiting the toilet, feeding, medications and or medication intake, moving indoors and outdoors, walking, oral care, oversight and other. The questions were constructed after processing the literature and discussing the questions with colleagues at a seminar. The structured interview guide is available in Swedish from the authors upon request. 
Third, to collect information about participants' utilization of health and social care, care records were collected for all patients in municipal care, in-patient care (emergency visit at a hospital, admitted as an in-patient or observational patient, always specialist care), out-patient care (primary care, health care centre, home visits, sometimes specialist care) and municipal care records were collected. All patient records were collected from the health centres (those written by physicians, district and registered nurses, physiotherapists and occupational therapists) and, in-patient's specialist care records. Documentation from municipal elderly care was collected in the form of needs assessments, decisions about service provision, needs assessment documentation and home service records. Data were collected about the numbers of enrolled nurses who had visited the participants and the time intervals between their visits. Economists from the county councils and the communities agreed on a common cost calculation for health and social care utilization.

Fourth, all costs were collected from economists in in-patient care, out-patient care and municipal care. Informal carers were asked to remember the latest month during the 18-month period, since there is risk in asking questions that require longer-term memories [19]. The monthly costs were then multiplied by 18 to obtain the costs for the 18-month period. Costs of informal carers were calculated by the economist members of the study team based on template calculations for home service. For example, if an informal caregiver stated that it took two hours to cook food, that time was used for home help computations.

\section{Data analysis}

Statistical Package for the Social Sciences (SPSS) for Windows version 17.0 (SPSS Inc., Chicago, IL, USA) was used for data analysis. The majority of variables were measured on a ratio scale (for example age, hours) and some variables measured on a nominal scale (for example gender, living alone or not). The data were both non-parametric and parametric [20].

\section{Ethical approval and consent procedures}

Ethical approval was received from the Regional Board of Research Ethics, Uppsala, Sweden (Dnr 2010/099). The hospital manager in the region gave permission to access the patient register, patient records and costs. The director of elderly care and the first line manager in the municipal gave permission to access patients' needs assessments and home service. Participants and their relatives whom were interviewed gave their informed consent. Before giving informed consent, each was provided with information about the study, including that participation was voluntary and that the relatives could end their interview at any time without giving any reason. They were guaranteed confidentiality and anonymity in the presentation of results.

\section{Results}

\section{Characteristics of the participants and informal carers}

The participants $(n=20)$ were 11 men and 9 women, with an average age of 79 years ( $\min -\max =76-83$ years). They had an average of 4.5 medical diagnoses (min-max $=3-9$ ) and lived either alone (n $=9$ ) or with their spouses $(n=11)$. Of the 20 participants, 12 had informal carers, nine women and three men, with an average age of 67 years $(\min -\max =43-90)$. The informal carers were spouses $(n=5)$ (note that not all spouses were informal carers), children $(\mathrm{n}=5)$, a close friend $(n=1)$, or a sister-in-law $(n=1)$. Most of the participants $(n=12)$ were either married or cohabiting; the others were either unmarried $(n=1)$, divorced $(n=2)$ or a widow or widower $(n=5)$. Four of the informal carers were gainfully employed and nine were retired. Three of the four who were gainfully employed had active dependents who took time off from work to help their family member for 1-12 hours per month.

\section{Health, social and informal care costs}

Costs for the participants' health, social and informal care utilization during the 18-month period ranged from 12,152 to 137,959 Euros. According to the cost classification (high, medium low costs), by Swedish Association of Local Authorities and Regions (21), the results in table 1 reveal that seven participants had high costs, from 53,400 to 128,160 Euros, seven had medium costs, from 26,700 to 53,293 Euros and six had low costs, from 3397 to 26,593 Euros. The highest health care costs for all 20 participants were specialist care during in-patient care. The calculated costs for informal care ranged from 2092 to 70,590 Euros.

\section{Health, social and informal care utilization}

The twenty participants had an average of $19.5(\min -\max =7-95)$ in-patient care days and 5 (min-max $=3-17$ ) care episodes during the 18 -month period. A care episode was defined as a continuous period between in-patient care registration and discharge. Care episodes could be divided into several hospital admissions at various clinics and/or hospitals which were linked in time. The participants had an average of 4 (min-max $=1-7)$ emergency visits and took during the study period an average of 10.5 (min-max $=2-27$ ) medications, of which 1.6 were prescribed if necessary. The participants received specialist care in out-patient care through an average of 6.5 (min-max $=$ $1-35)$ physicians visits and $5.5(\min -\max =1-45)$ district nurses visits. They also had an average of $5(\min -\max =1-15)$ physician visits and $6(\min -\max =1-27)$ district nurse visits at primary care health care centres. Eight participants had an average of 14.5 (min-max $=8-141)$ district nurse home visits. The number of decisions about service provision averaged $2(\min -\max =1-5)$ during the 18 -month period. Those with home service received visits from enrolled nurses and nursing aids 0-7 times per week. Ten participants had municipal home service and were granted the maximum time for home service (an average of 206 hours during a month), although they only used an average of 125 hours.

Twelve participants had relatives who were involved in their health and social care. These relatives provided informal care for an average of 8 (min-max $=1-68$ ) hours weekly during the 18-month period. Those relatives who lived with the participants provided the highest number of hours of care. The most common chores performed by the informal carers were grocery shopping, cooking and washing clothes, which were performed by eight informal carers. They also assisted the participants with morning and evening routines including feeding, cleaning, showering, oral health care, dressing, undressing, toilet visits, medications, moving indoors and outdoors and finances.

\section{Discussion}

\section{Discussion of the results}

The results describe an overall picture of the health, social and informal care utilization and costs of participants. The answers to 
questions about humans' daily living are often in such information. This information show that utilization of health and social care was from different principals with a focus on health problems. In addition, Akner stressed that care for older adults with multimorbidity is not sufficiently proactive in preventing multimorbidity or avoiding emergency care [21]. Earlier research argued that care should not focus on health problems, but rather on providing co-ordinated management [22]. Policy changes in Swedish health care and social service laws have led to a polarization of health care and social care, with each counteracting the other's aims and, thereby, contributing to unclear accountability between organizations [13]. Political steering, leadership and cooperation need to work well to promote availability and continuity in health and social care management for older adults with multimorbidity [13].

According to previous research one way of intervening in a health system that is providing fragmented care is to utilizing Case management, in other words models that are used to co-ordinate care for people with complex needs, such as older adults with multi-morbidity [23]. It is widely recognized that patient-centered care is important and that caring is conducted in a moral manner respecting patients' dignity and their trust $[24,25]$. At the same time, while a patient-centered approach is retained, the findings of this study could increase care staff's ability to see the cost and organization of health and social care. Registered nurses well equipped to act based on their holistic view of the patient. Thus, it is important to increase registered nurses' influence on decisions made at work [26].

This study shows that participants' health, social and informal care utilization costs during the 18-month period ranged from 12,084 to 137,187 Euros. This cost distribution is consistent with the national figures in Sweden and is in line with the findings of several studies demonstrating an association between multimorbidity and high health care costs and associated costs across the health care system $[2,8,9,22]$. This study showed that the highest health care costs for all participants were from utilization of specialist care within in-patient care. This might not be surprising, since in-patient care is known to be more expensive than out-patient care. The reason for this is a primary care system with strong gate keeping that is associated with high healthcare utilization and costs across the healthcare system [10]. The term gatekeeping is used to denote the complex situation "to guard the money or assess the needs of older people" [27]. The gatekeeper situation makes it difficult to make the necessary decisions in accordance with an individual's needs assessment. Tanenbaum argues that when using one of four models, that is, the model focusing on patients, providers and states versus 'the system', it can be used to focus on goals other than the patient, for example, reduced costs [28]. Our own reflection is that a successful health and social care system should focus on the patient, not the budget and costs, since doing so might actually be more cost-effective. However, there is some criticism that it is impossible to obtain precise information about what is meant by "cost-effectiveness". Carlsson et al., discuss what the limits should be and how much society is willing to invest in quality-adjusted life years [29].

This study reveals informal care in daily living for older adults with multimorbidity. We speculate that an older adult's condition may, at times, change quickly and, therefore, that those with multimorbidity rely on help from relatives when the need occurs. This study's results show the costs of informal care and how such care is cost-effective, especially for principal municipal authorities responsible for care in homes for older adults. But that this is not a sustainable solution. The next step should be to unwind the knowledge of the clinical effects of knowledge about patient benefits in relation to costs [29]. Participants' relatives played a crucial role, providing informal care for between 1 and 68 hours weekly, with the calculated costs for informal care reaching as much as 70,590 Euros during the 18-month period. High levels of informal care responsibility are associated with poorer health and pointed out that informal carers spend more time caring for others than they do caring for their own health [10].

Consequently, support for informal carers is an important resource for older adults with multimorbidity $[10,13]$. It is important that respite care be available for informal carers who need relief from what is often intensive round-the-clock work. Thus, health and social care management should include responding well to informal carers and offering them support so that they feel secure and are able to cope with their situation and to reduce their risk of ill health. If informal carers cannot manage their situation, the health and social care systems may face collapse [11]. It is essential to organize health care delivery systems to improve health outcomes and health care management to address chronic disease management. Therefore, providing individualized, comprehensive and coordinated management in a well-planned sequence without unnecessary duplication of effort is a matter of urgency [21,30,31]. A comprehensive approach, which includes social, cognitive and functional problems and diseases, is needed. One approach is the Comprehensive Geriatric Assessment (CGA), which assesses older adults with multimorbidity. The CGA allows a specific care plan for each individual patient. Studies have shown that the CGA, in association with an integrated team of geriatric physicians, registered nurses, social workers and other professionals assessing and managing the healthcare problems identified by the CGA, together with individualized care plans have resulted in improved care planning and better quality of care [21,30,31]. This approach might improve older adults' health and rationalize the use of resources [21,30,31].

\section{Discussion of the methods}

The study has been performed in 2011 and is retrospective in nature. In 1992, elderly care was restructuring in Sweden [2,32]. This restructuring still exists and should not decrease the validity of data in this study for today's healthcare issues. The major strength of this study is that it provides information over an 18-month period about a relatively small sample of 20 older adults to reach an in-depth understanding of their daily lives. This was a random sample in a restricted area; however, generalizability of the findings to other populations is limited. Nevertheless, the results are important and are relevant to clinical practice. The choice of inclusion criteria was in accordance with the Swedish version of the International Statistical Classification of Diseases and Related Health Problems ICD-10-SE [7]. This might increase reliability. Informal carers were asked to describe the help and care they provided during the last month of the study period. Self-reported information may have affected reliability. Then, the costs of this informal care were multiplied by 18 to arrive at an overall cost for the 18-month period and there might be a weakness in the calculations for informal care. This may have affected reliability. On the other hand, there is a risk in asking questions that rely on long-term memory for responses [20]. 
Citation: Josefsson K, Meranius MS (2018) Complexity in Daily Living of Older Adults with Multimorbidity: Health, Social and Informal Care Utilization and Costs. J Gerontol Geriatr Med 4: 017.

\begin{tabular}{|c|c|c|c|c|c|c|c|c|c|}
\hline \multirow[t]{3}{*}{ Gender } & \multirow[t]{3}{*}{ Age } & \multirow[t]{3}{*}{ Living alone } & \multirow[t]{3}{*}{ Total costs } & \multicolumn{3}{|c|}{ County council } & \multirow[t]{3}{*}{$\begin{array}{l}\text { Total care } \\
\text { costs }\end{array}$} & \multirow{3}{*}{$\begin{array}{c}\text { Municipal } \\
\text { ome service (social care), } \\
\text { short-term housing }\end{array}$} & \multirow{3}{*}{$\begin{array}{l}\text { Informal } \\
\text { care }\end{array}$} \\
\hline & & & & \multirow{2}{*}{$\begin{array}{l}\text { In-patient care } \\
\text { Specialist care }\end{array}$} & \multicolumn{2}{|c|}{ Out-patient care } & & & \\
\hline & & & & & $\begin{array}{l}\text { Specialist } \\
\text { care }\end{array}$ & $\begin{array}{l}\text { Primary care: Health } \\
\text { care centres }\end{array}$ & & & \\
\hline & & Yes/No & Euros & Euros & Euros & Euros & Euros & Euros & Euros \\
\hline Female & 83 & Yes & 111,553 & 55,354 & 8028 & 3393 & 66,775 & 37,431 & 7346 \\
\hline Male & 78 & No & 69,834 & 32,826 & 1769 & 10,056 & 44,651 & 10,056 & 14,838 \\
\hline Male & 76 & No & 137,959 & 101,041 & 8130 & 1508 & 110,679 & 0 & 26,706 \\
\hline Male & 80 & No & 72,404 & 23,912 & 19,587 & 326 & 43,825 & 0 & 28,277 \\
\hline Female & 79 & Yes & 23,397 & 10,006 & 4803 & 630 & 15,439 & 7860 & 0 \\
\hline Female & 79 & Yes & 54,785 & 6596 & 13,886 & 3743 & 24,225 & 7015 & 3317 \\
\hline Male & 78 & No & 24,961 & 15,345 & 7754 & 1758 & 24,857 & 0 & 0 \\
\hline Female & 81 & No & 18,080 & 10,693 & 6448 & 865 & 18,006 & 0 & 0 \\
\hline Male & 80 & No & 40,365 & 28,341 & 10,148 & 1708 & 40,197 & 0 & 0 \\
\hline Female & 81 & Yes & 86,323 & 46,244 & 4183 & 2011 & 52,438 & 29,338 & 4189 \\
\hline Male & 78 & No & 54,042 & 21,419 & 3845 & 276 & 25,540 & 0 & 28,277 \\
\hline Male & 80 & Yes & 55,225 & 39,948 & 6287 & 3031 & 49,266 & 5729 & 0 \\
\hline Female & 83 & Yes & 99,709 & 67,762 & 13,523 & 2983 & 84,268 & 1934 & 13,091 \\
\hline Female & 79 & No & 34,915 & 18,976 & 5240 & 5376 & 29,592 & 988 & 4189 \\
\hline Male & 77 & No & 23,888 & 15,061 & 8028 & 703 & 23,792 & 0 & 0 \\
\hline Male & 78 & No & 12,152 & 6071 & 4233 & 1805 & 12,109 & 0 & 0 \\
\hline Male & 82 & Yes & 23,719 & 8217 & 4285 & 648 & 13,150 & 0 & 10,473 \\
\hline Female & 82 & No & 98,422 & 13,111 & 4137 & 9147 & 26,395 & 964 & 70,692 \\
\hline Female & 78 & Yes & 34,349 & 16,061 & 5629 & 1893 & 23,583 & 10,630 & 0 \\
\hline \multirow[t]{2}{*}{ Male } & 76 & Yes & 70,656 & 27,033 & 7400 & 4327 & 38,760 & 29,526 & 2095 \\
\hline & & & 1142,430 & 584,018 & 147,342 & 56,110 & 767,547 & 141,470 & 205,196 \\
\hline
\end{tabular}

Table 1: Participants' health, social and informal care utilization and costs during an 18-month period.

\section{Conclusion}

Older adults' daily living includes many health and social care contacts, from different principals, different care levels and different staff. Therefore, given the increasing number of older adults with multimorbidity health, social care utilization and costs, a holistic view and a comprehensive approach should be considered when healthcare policy, the organization of health and social care are discussed. This study could be used to enable care staff to widen their horizons and see the cost and organization of health and social care while retaining a patient-centered approach.

\section{Conflict of Interest}

There was no conflict of interest that might influence the results or interpretation of the results.

\section{Acknowledgments}

We are grateful to all of the study participants. The research was supported by Västmanl and Association of Local Authorities and County Council, Sweden.

\section{References}

1. Department of Economic and Social Affairs (2002) World Population Ageing: 1950-2050. Department of Economic and Social Affairs, Population Division, United Nations, New York, USA.

2. Anell A, Glenngård AH, Merkur S (2012) Sweden health system review. Health Syst Transit 14: 1-159.

3. Smith SM, Wallace E, O'Dowd T, Fortin M (2012) Interventions for improving outcomes in patients with multimorbidity in primary care and community settings. Cochrane Database Syst Rev 4: CD006560.

4. Chi MJ, Lee CY, Wu SC (2011) Multiple morbidity combinations impact on medical expenditures among older adults. Arch Gerontol Geriatr 52: 210-214.

5. Orueta JF, García-Álvarez A, García-Goñi M, Paolucci F, Nuño-Solinís R (2014) Prevalence and costs of multimorbidity by deprivation levels in the basque country: a population based study using health administrative databases. PLoS One 9: 89787.

6. Marengoni A, Angleman S, Melis R, Mangialasche F, Karp A, et al. (2011) Aging with multimorbidity: a systematic review of the literature. Ageing Res Rev 10: 430-439.

7. WHO (2015) ICD-10 online versions. ICD, WHO, Geneva, Switzerland. 
8. Yoon J, Zulman D, Scott JY, Maciejewski ML (2014) Costs associated with multimorbidity among VA patients. Med Care 52: 31-36.

9. Glynn LG, Valderas JM, Healy P, Burke E, Newell J, et al. (2011) The prevalence of multimorbidity in primary care and its effect on health care utilization and cost. Fam Pract 28: 516-523.

10. Jowsey T, McRae I, Gillespie J, Banfield M, Laurann Y (2013) Time to care? Health of informal older carers and time spent on health related activities: an Australian survey. BMC Public Health 13: 374.

11. Josefsson K (2010) Tio framgångsfaktorer för en god och säker hemsjukvård för äldre personer [Ten success factors for a good and safe home care for older people]. Swedish Nursing Association and Careers Association, Stockholm, Sweden. Pg no: 20.

12. Davey A, Malmberg B, Sundström G (2013) Aging in Sweden: local variation, local control. Gerontologist 54: 525-532.

13. Meranius MS, Josefsson K (2017) Health and social care management for older adults with multimorbidity: a multiperspective approach. Scand J Caring Sci 31: 96-103.

14. Gill A, Kuluski K, Jaakkimainen L, Naganathan G, Upshur R, et al. (2014) "Where do we go from here?" Health system frustrations expressed by patients with multimorbidity, their caregivers and family physicians. Health Policy 9: 73-89.

15. Bratzke LC, Muehrer RJ, Kehl KA, Lee KS, Ward EC, et al. (2015) Self-management priority setting and decision-making in adults with multimorbidity: A narrative review of literature. Int J Nurs Stud 52: 744-755.

16. Nordenfelt L (1991) Livskvalitet och hälsa: teori \& kritik [Quality of life: Theory \& criticism]. Almqvist \& Wiksell, Sweden. Pg no: 160.

17. Polit D, Beck C (2011) Nursing Research: Generating and Assessing Evidence for Nursing Practice (9thedn). Lippincott Williams \& Wilkins, Pennsylvania, United States.

18. Gurner U (2000) Multiskröpligas vardag - att se helheten [Frail elderlys' everyday life - to see the whole picture]. Stiftelsen Stockholms läns Äldrecentrum, Stockholm, Sweden.

19. Schaeffer NC, Presser S (2003) The Science of Asking Questions. Annual Review of Sociology 29: 65-88.

20. Altman DG (1990) Practical Statistics for Medical Research. Chapman \& Hall, CRC Press, Florida, USA.

21. Akner G (2013) Frailty and Multimorbidity in Elderly People: A Shift in Management Approach. Clinical Geriatrics 21.
22. SALAR, Swedish Association of Local Authorities and Regions (2012) Utanför sjukhuset. Kvalitativ uppföljning av multisjuka äldre i ordinärt boende [Outsidethe hospital. Qualitative monitoring of older adults with multimorbidity in regular housing]. Swedish Association of Local Authorities and Regions, Stockholm, Sweden.

23. Gustafsson M, Kristensson J, Holst G, Willman A, Bohman D (2013) Case managers for older persons with multi-morbidity and their everyday work - a focused ethnography. BMC Health Serv Res 13: 496.

24. Kvåle K, Bondevik M (2008) What is important for patient centred care? A qualitative study about the perceptions of patients with cancer. Scand $\mathrm{J}$ Caring Sci 22: 582-589.

25. Slettebø Å, Fredriksson L (2015) The significance of caring. Scand J Caring Sci 29: 203-204.

26. Josefsson K, Sonde L, Winblad B, Robins Wahlin TB (2007) Work situation of registered nurses in municipal elderly care in Sweden: A questionnaire survey. Int J Nurs Stud 44: 71-82.

27. Norman E (2010) Biståndshandläggare - att vakta pengar eller bedöma äldres behov [Assistance officer - to guard the money or assess the needs of older people]. Stiftelsen Stockholms läns Äldrecentrum, Gävlegatan, Stockholm, Sweden.

28. Tanenbaum SJ (2015) What is Patient-Centered Care? A Typology of Models and Missions. Health Care Anal 23: 272-287.

29. Carlsson P, Anell A, Eliasson M (2006) Hälsoekonomi får allt större roll för sjukvårdens prioriteringar [Health economicsis of increasingrole inhealth carepriorities]. Läkartidningen 46: 3617-3624.

30. Ellis G, Whitehead MA, Robinson D, O'Neill D, Langhorne P (2011) Comprehensive geriatric assessment for older adults admitted to hospital: meta-analysis of randomised controlled trials. BMJ 27: 343.

31. Onder G, Palmer K, Navickas R, Jurevičienè E, Mammarella F, et al. (2015) Time to face the challenge of multimorbidity. A European perspective from the joint action on chronic diseases and promoting healthy ageing across the life cycle (JA-CHRODIS). Eur J Intern Med 26: 157-159.

32. National Board of Health and Welfare (1996) The "Ädel Reform" Final Report 1996 English summary in: Ädelreformen. Slutrapport. Socialstyrelsen följer upp och utvärderar. National Board of Health and Welfare, Stockholm, Sweden. Pg no: 85-88. 